\title{
RECOGNIZABILITY OF HEXAGONAL PICTURE LANGUAGES BY HEXAGONAL WANG AUTOMATA
}

\author{
Kamaraj T. ${ }^{1}$, Thomas D.G. ${ }^{2}$, Kalyani T. $^{3}$ \\ ${ }^{1}$ Department of Mathematics, Sathyabama University, Chennai, India \\ ${ }^{2}$ Department of Mathematics, Madras Christian College, Chennai, India \\ ${ }^{3}$ Department of Mathematics, St. Joseph's College of Engineering, Chennai, India \\ Email: 1'kamaraj_mx@yahoo.co.in
}

\begin{abstract}
Here we present an automata model for the recognizability of hexagonal picture languages, called Hexagonal Wang automata (HWA), which is based on a variant of hexagonal Wang tiles. We provide wide range of polite scanning strategies and prove that the non deterministic HWA with any polite scanning strategy are equivalent to Hexagonal Tiling Systems or Hexagonal online Tessellation Acceptors. We also introduce the notion of deterministic recognizability in HWA and present some comparison results.
\end{abstract}

Key words: Hexagonal picture languages, hexagonal Wang system, scanning strategy, determinism.

\section{INTRODUCTION}

Hexagonal arrays generated by grammars or recognized by automata are found in the literature in the framework of picture processing and pattern recognition tasks [1,4]. In [1], the authors introduced the notion of hexagonal tiling systems and hexagonal Wang systems and showed the equivalence between the two. This provided new formalisms to recognize hexagonal picture languages. In [2], the authors introduced, Wang automata, a model of automaton for rectangular picture language recognition, which is based on Wang tiles. Wang automata combined the features of both online tessellation automata and 4-way automata. It is natural to consider the hexagonal version of Wang automata for the recognition of hexagonal picture languages. In [5], the authors presented such model with few polite scanning strategies which sorted all positions in a hexagonal picture and does not depend on the input symbols. Now we introduce Hexagonal Wang automata (HWA) directed by a wide range of the polite scanning strategies and prove that this model, in non deterministic form, is equivalent to hexagonal tiling systems or hexagonal online tessellation automata. We also introduce a natural notion of determinism in HWA and compare some of the deterministic classes of HWA.

The paper is organized in the following manner. In section 2, we recall some basic notions of hexagonal pictures and hexagonal picture languages, hexagonal tiling and Wang systems. In section 3 , we introduce a wide range of polite scanning strategies for hexagonal pictures. In section 4, we present a hexagonal version of Wang automaton named as Hexagonal Wang Automata (HWA) and prove a characterizing theorem relating to HREC. In section 5, deterministic HWA is introduced and some comparisons have been made among the deterministic classes of HWA.

\section{PRELIMINARIES}

Let $\Sigma$ be a finite alphabet of symbols. A hexagonal picture $p$ over $\Sigma$ is a hexagonal array of symbols of $\Sigma$. A p-hexagonal array is a six-sided convex hexagonal array whose opposite sides are parallel. A $b$-hexagonal array is an equiangular $p$-hexagonal array whose opposite sides are equal. $\Sigma^{* *} \mathrm{H}$ denote the set of all $b$-hexagonal arrays over the symbols alphabet $\Sigma$. With respect to a triad of triangular axes $x, y, z$. i.e.,

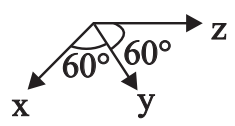

the coordinates of each element of a hexagonal picture can be fixed. Figure 1 represents the coordinates of the hexagonal picture

$a{ }_{b}^{a} b^{a} a$


$\Sigma^{1, m, n}$ denotes the set of hexagonal pictures of size $(I, m, n)$ i.e., having $I$ elements on the side along $x$ axis, $m$ elements on the side along $y$ axis and $n$ elements on the side along $z$ axis.

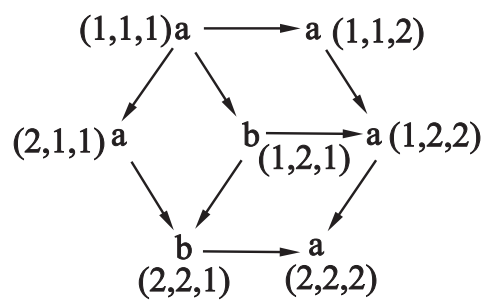

Fig. 1 Coordinates of a Hexagonal picture

\section{Example 2.1}

A hexagonal picture $p \in \Sigma^{2,3,4}$ over the alphabet

$\Sigma=\{a, b, c\}$ is shown in Figure 2.

$$
\begin{aligned}
& \begin{array}{llll}
a & b & b & c
\end{array} \\
& \begin{array}{c}
\mathrm{b} \underset{\mathrm{a}}{\mathrm{b}} \mathrm{c} \mathrm{c}^{\mathrm{b}} \mathrm{a}^{\mathrm{b}} \mathrm{b} \\
\mathrm{b}
\end{array}
\end{aligned}
$$

Fig. 2 A hexagonal picture of size $(2,3,4)$

\section{Definition 2.1}

If $p \in \Sigma^{* *}$ then $\hat{p}$ is the hexagonal array obtained by surrounding $p$ with a special boundary

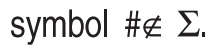

\section{Example 2.2}

For the picture $p$ given in example $1, \hat{p}$ is given in Figure 3.

$$
\begin{aligned}
& \text { \# \# \# \# \# } \\
& { }^{\#}{ }^{\mathrm{b}}{ }^{\mathrm{a}} \mathrm{a}^{\mathrm{b}} \mathrm{c}^{\mathrm{b}} \mathrm{b}^{\mathrm{c}} \mathrm{b}^{\#}
\end{aligned}
$$

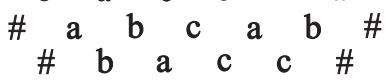

$$
\begin{aligned}
& \text { \# \# \# \# \# }
\end{aligned}
$$

Fig. 3 Hexagonal picture bordered with \#

\section{Definition 2.2}

A pixel is an element $p(i, j, k)$ of $p$. We call $(i, j, k)$, the position in $p$ of the pixel. Picture domain (or domain), denoted by dom $(p)$, is to refer to the set of possible positions in a hexagonal picture of size $(I, m, n)$. Each position has six edges.
Given a hexagonal picture $p$ of size $(I, m, n)$ for $g \leq l, h \leq m$ and $k \leq n$, we denote by $\mathrm{B}_{\mathrm{g}, h, \mathrm{k}}(\mathrm{p})$ the set of all hexagonal sub pictures of $p$ of size $(g, h, k)$. Each member of $B_{2,2,2}(p)$ is called a hexagonal tile. For example, $a_{b}{ }_{b}{ }_{a}^{a} a^{*}$ is a hexagonal tile with two symbols. Let $\Gamma$ and $\Sigma$ be two finite alphabets, let $p \in \Gamma^{* *} \mathrm{H}$.

\section{Definition 2.3}

The projection by mapping $\pi: \Gamma \rightarrow \Sigma$ of picture $p$ is the picture $p^{\prime} \in \Sigma^{\star * H}$ such that $p^{\prime}(i, j, k)=\pi(p(i, j, k))$ for all $1 \leq i \leq l, 1 \leq j \leq m$ and $1 \leq k \leq n$, where $(I, m, n)$ is the size of the hexagonal picture.

\section{Definition 2.4}

Let $\Gamma$ be a finite alphabet. A hexagonal picture language $L \subseteq \Gamma^{* *} \mathrm{H}$ is called local if there exists a finite set $\Delta$ of hexagonal tiles over $\Gamma \cup\{\#\}$ such that $L=\left\{p \in \Gamma^{* * H} / B_{2,2,2}(\bar{p}) \subseteq \Delta\right\}$.

The family of local hexagonal picture languages will be denoted by HLOC.

\section{Definition 2.5}

A hexagonal picture language $L \subseteq \Sigma^{* *} H$ is called recognizable if there exists a local hexagonal picture language $L^{\prime}$ over an alphabet $\Gamma$ and a mapping $\pi: \Gamma \rightarrow \Sigma$ such that $L=\pi\left(L^{\prime}\right)$.

The family of recognizable hexagonal picture languages will be denoted by HREC.

\section{Definition 2.6}

A hexagonal tiling system $T$ is a 4-tuple $\langle\Sigma, \Gamma, \pi, \theta\rangle$ where $\Sigma$ and $\Gamma$ are two finite set of symbols, $\pi: \Gamma \rightarrow \Sigma$ is a projection and $\theta$ is a set of hexagonal tiles over the alphabet $\Gamma \cup\{\#\}$.

\section{Definition 2.7}

A hexagonal picture language $L \subseteq \Gamma^{* * H}$ is hexagonal tiling recognizable if there exists a hexagonal tiling system $T=\langle\Sigma, \Gamma, \pi, \theta\rangle$ such that $=\pi(L(\theta))$.

The family of hexagonal tiling recognizable languages is denoted by $L$ (HTS). It is easy to see that HREC is exactly L (HTS). 


\section{Example 2.3}

Consider the language $L_{x \text {-parallel }}$ of hexagonal pictures over $\Sigma=\{a, b\}$ with the opposite borders in the $x$-direction (parallel borders) have identical elements. Let $\Gamma$ be the set of symbols of the form $k_{t}$ with $k \in \Sigma, t \in \Sigma \cup\{\bullet\}$. For each picture in $\mathrm{L}_{\mathrm{x} \text {-parallel }}$ consider the picture $p^{\prime} \in \Gamma^{* *}$, where subscripts are used to connect corresponding identical symbols in the parallel borders along L-like paths. The following is an example of such pair of hexagonal pictures $p$ and $p^{\prime}$.

$$
\begin{aligned}
& p={ }_{a}^{a}{ }_{b}^{a} c^{b} c^{b} b^{a} a
\end{aligned}
$$

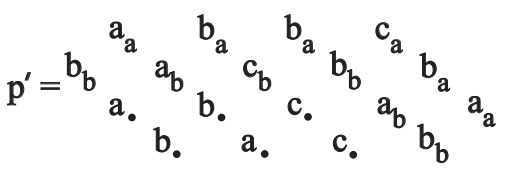

It can be easily proved that the language of $p^{\prime}$ is local and hence $L_{x \text {-parallel }}$ is in HREC.

In [1], the authors used hexagonal Wang tiles to introduce Hexagonal Wang Systems (HWS), a formalism to recognize hexagonal picture languages. The authors also proved the equivalence of HWS and HTS. Here we give definitions by using the equivalent notations introduced in [5].

\section{Definition 2.8}

A labeled hexagonal Wang tile is a 7-tuple consisting of six colours chosen from a finite set of colours $Q$ and a label from a finite alphabet $\Sigma$. The colours are placed at upper left (UL), upper right (UR), left $(L)$, right $(R)$, lower left $(L L)$, lower right $(L R)$ positions of the label as in Figure 4.

$$
\text { LL label } R
$$

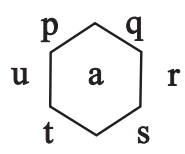

Fig. 4 Labeled hexagonal Wang tile

Two hexagonal Wang tiles may be adjacent if and only if the adjacent edges are of same colour. In the rest of the paper, it is supposed that the hexagonal Wang tiling cover the plane, so that all the tiles that are beyond the borders of the considered picture will be special tile $B=($ \#, \#, \#, \#, \#, \#, \# ).

\section{Definition 2.9}

For any direction $d \in$ Dirs $^{\mathrm{H}}=\{\measuredangle, \nearrow$, $\leftarrow, \rightarrow, \searrow, \backslash\}, A_{d}$ is the colour of the edge of a hexagonal tile $A$ towards the direction $d .-d$ refers to the direction opposite to $d$. $\lambda(A)$ refers to the label of the hexagonal tile $A$. In case of $A$ given in Figure 1,

$$
\begin{aligned}
& A \nwarrow=p, A_{\rightarrow}=r, A_{\swarrow}=t, A \nearrow=q, A \longleftarrow=u, A \searrow=s \\
& \text { and } \lambda(A)=a \text {. }
\end{aligned}
$$

The set of tiles with labels in $\Sigma$ and colours in $Q$ is denoted by $\Sigma_{6 Q}$.

\section{Definition 2.10}

Partial hexagonal tiles are tiles where some colours are not defined. The set of partial tiles is denoted by $\Sigma_{Q}$. Domain of a tile $A$ is the set $\Delta_{A}$ of directions where $A$ is defined.

\section{Definition 2.11}

Given two partial hexagonal tiles $A, C$ bearing the same label, $C$ extends $A$ if $C_{d}=A_{d}$ for every $d \in \Delta_{A}$. A hexagonal tile which is not partial can be called as complete.

Labeled hexagonal Wang tiles in $\Sigma_{6 Q}$ can be used to build hexagonal pictures over $\Sigma$.

\section{Definition 2.12 Hexagonal Wang pictures}

A hexagonal picture $P \in \Sigma_{6 Q}^{* * H}$ is called an hexagonal Wang picture, if all borders are coloured with \# and two tiles may be adjacent only if the colour of the touching edges is the same.

We see in Figure 5, an example of a hexagonal Wang picture of size $(2,2,2)$, where we represent common colour only once between the adjacent edges.

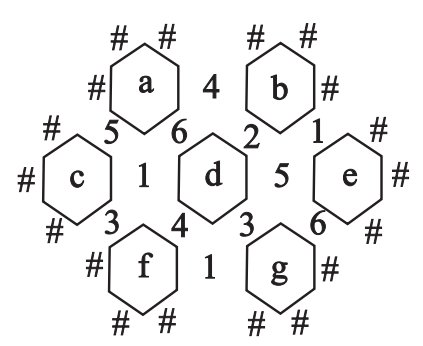

Fig. 5 A hexagonal Wang picture of size $(2,2,2)$ 


\section{Definition 2.13}

The label of a hexagonal Wang picture $P$ over $\Sigma_{6 \mathrm{Q}}$ is the picture $p=\lambda(P) \in \Sigma^{* * \mathrm{H}}$ having the labels of pixels of $P$ as the elements of $p$. i.e., $p(i, j, k)=(P(i, j, k))$. For example the label of the above Wang picture is ${ }_{c}{ }_{f}^{a}{ }_{g}{ }_{g}$ e.*

\section{Definition 2.14}

Hexagonal Wang fragments are the picture fragments whose pixels are partial hexagonal tiles with compatible borders. For example, if

$$
\mathrm{A}=1 \underbrace{\mathrm{a}]_{\#}^{\#}}_{4} 3, \quad \mathrm{C}=3 \overbrace{\mathrm{c}]_{\#}^{\#}}^{\#} 4, \mathrm{~B}=\overbrace{\#}^{\#} \# \text {, }
$$

$\mathrm{AC}$ is the following fragment $\mathrm{B}$

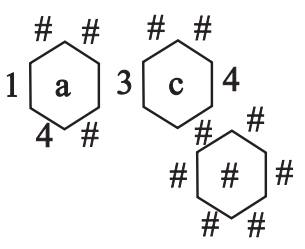

\section{Definition 2.15}

A hexagonal Wang system is a triple $W=\langle\Sigma, Q, \theta\rangle$, where $\Sigma$ is a finite alphabet, $Q$ is a set of colours, $\theta$ is a subset of $\Sigma_{6 Q}$. The language generated by $W$ is the language, $L(W) \wp \Sigma^{* *}$ of the labels of all hexagonal Wang pictures over $\theta$. HREC is the class of picture languages generated by Hexagonal Wang systems [1].

\section{Example 2.4}

The language $L_{b \text {-centric }}$ of hexagonal pictures of equal size on all sides over $\{a, b\}$, having $b$ only at the center is generated by the Wang system $<\Sigma, Q, \theta>$ where $Q=\{-, \Lambda, \bullet, \#\}$ and is the set of

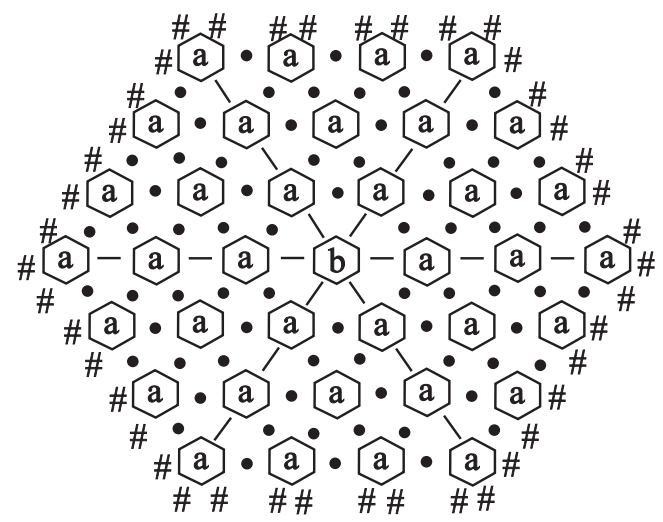

Fig. 6 Set of Hexagonal Wang Tiles hexagonal Wang tiles contained in the picture $P$ in the Figure 6.

\section{HEXAGONAL SCANNING STRATEGIES}

Hexagonal scanning strategies for hexagonal pictures are discussed in [5]. The authors considered strategies that do not depend on the content of the input picture. Scanning strategies are defined in terms of partial functions; for a partial function $g$, if the value of $g$ at $t$ is not defined, that $g(t)=\perp$. We now recall these strategies along with some new ones and provide examples.

\section{Definition 3.1}

A hexagonal scanning strategy [5] is a family, $\delta=\left\{\delta_{t \times m \times n}:\{1,2, \ldots\} \rightarrow \operatorname{dom}(p), p \in \Sigma^{(l, m, n) H}\right\}$ where each $\delta_{t \times m \times n}$ is a partial function such that $\delta_{t \times m \times n}(t) \neq \perp$ for some $t$ implies $\delta_{t \times m \times n}(s) \neq \perp$ for every $1 \leq s \leq t, \delta_{t \times m \times n}$ is called the hexagonal scanning function over the hexagonal picture domain $I \times m \times n$. A scanning strategy is said to be continuous if for every $t, I, m, n, \delta_{t \times m \times n}(t+1)$ is adjacent to $\delta_{t \times m \times n}(t)$, provided they are both defined.

\section{Definition 3.2}

A hexagonal scanning strategy is said to be one-pass if each scanning function $\delta_{t \times m \times n}$ restricted to $\{1,2, \ldots,|\operatorname{ldom}(p)|\}$ where $p \in \Sigma^{(l, m, n) H}$, is a bijection and $\delta_{t \times m \times n}(t)=\perp$ for every $t>|\operatorname{dom}(p)|$.

A hexagonal strategy provides a method to visit positions in any hexagonal picture domain: $\mu_{t \times m \times n}(t)$ is the position visited in dom (p) at time $t$. One-past strategies are those that visit each position in each domain exactly once.

\section{Definition 3.3}

A hexagonal scanning strategy is said to be blind if it proceeds locally, by scanning adjacent positions. It cannot see neither hexagonal picture context nor its size: it can only realize a border \# and an already considered position, when reaches it.

Some examples of one pass hexagonal scanning strategies with the picture size $3 \times 3 \times 4$ are given in Figure 7 (a-e) 


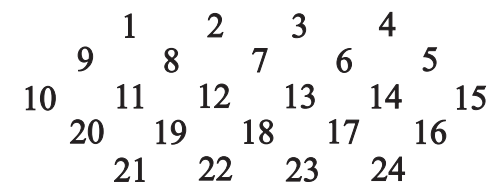

(a)

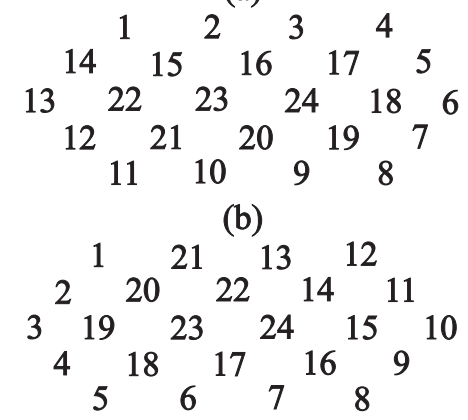

(c)

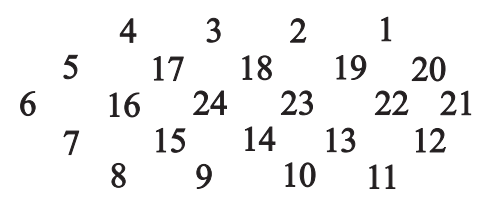

(d)

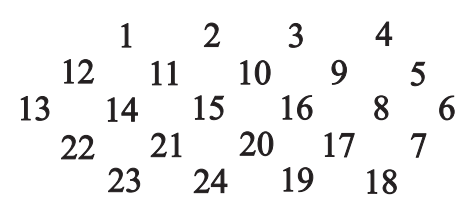

(e)

Fig. 7 Some one-pass hexagonal scanning strategies

The number in each pixel denotes its scanning order.

(a) has a snake like form $(\underset{\longrightarrow}{\rightleftharpoons})$

(b) has spiral form ( (a) )

(c) has nested pentagon form $(\iota)$

(d) has nested $\mathrm{C}$ like path $(\subset)$

(e) has arrow head like path $(\longrightarrow)$

These scanning strategies include the rotations of $n 60^{\circ}$ where $n=1,2,3,4,5,6$

The following notions add some constraints to the scanning strategies to implement blindness.

Given a position $x$, we denote by $\langle x\rangle$, the set of 6 edges adjacent to $x$ and $d \in$ Hdirs, the edge of $x$ in the direction $d$ is denoted by $x_{d}$ and the position adjacent to $x$ in direction $d$ is denoted by $x * d$.

\section{Definition 3.4}

A next-position function is a partial function $\eta: 2^{\text {Hdirs }} \times$ Hdirs $\rightarrow$ Hdirs such that $\eta(D, d)=\perp$ if $-d \notin D$. Here $\eta$ is used to choose where to go next: for a given position, there may be a set of already considered edges, given by the set $D$ of directions and $d$ is the direction from the last considered edge.

If $|D|=1$, then $d$ is the unique element of $D$, i.e., $d=-d$; if $|D|=5$, then $d$ is uniquely determined. If $1<|D|<5$, then $d$ may not be unique.

After fixing any next position function, any starting corner cor $_{s} \in$ corners $=\{u l, u r, I m, r m, I I, I r\}$ any starting direction $d_{S} \in$ Hdirs for every picture domain $1 \times m \times n$, consider the following hexagonal scanning function $\delta_{t \times m \times n}$ over $I \times m \times n$.

The starting position is

$$
\delta_{l \times m \times m}(I)=\left\{\begin{array}{l}
(1,1,1) \text { if } \text { cor }_{s}=u l \\
(1,1, n) \text { if } \cos _{s}=u r \\
(l, 1,1) \text { if } \text { cor }_{s}=\text { Im } \\
(1, m, n) \text { if } c o r_{s}=r m \\
(l, m, 1) \text { if } \text { cor }_{s}=\| \\
(l, m, n) \text { if } \text { cor }_{s}=I r
\end{array}\right.
$$

We also define $O_{1}$ as the set of outer edges (i.e., those adjacent to borders \#) of the picture domain $I \times m \times n$ and we set $d_{1}=d_{s}$.

The inductive definition of $\delta_{/ \times m \times n}(t+1)$ for $t \geq 1$ is given by:

$$
\begin{aligned}
& D_{t}=\left\{d \in H \text { dirs: }\left(\delta_{t \times m \times n}(t)\right) d \in O_{t}\right\} \\
& O_{t+1}=O_{r} \cup \text { edges }\left(\delta_{t \times m \times n}(t)\right) \\
& d_{t+1}=\eta\left(D_{t}, d_{t}\right) \\
& \delta_{t \times m \times n}(t+1)=\delta_{t \times m \times n}(t) * d_{t+1}
\end{aligned}
$$

Here we should notice that $\delta_{t \times m \times n}(1)-d_{1}$ must be in $O_{1}$ for $\eta\left(D_{1}, d_{1}\right)$ to be defined. 


\section{Definition 3.5}

A scanning strategy is blind if it is induced by a triple, $<\eta, \operatorname{cor}_{s}, d_{s}>$ where $\eta$ is a next position function, $\operatorname{cor}_{s}$ a starting corner and $d_{s}$ a starting direction.

\section{Definition 3.6}

A hexagonal scanning strategy is called polite if it is blind and one-pass. All the one-pass scanning strategies represented in Figure $7(a-d)$ are blind, hence polite. In particular we denote the strategies (a), (b), (c), (d) as

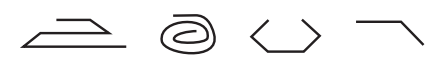

For example, $\longrightarrow$ is induced by the triple, $\langle\eta \neg, u l\rangle$,$\rangle where its next position function is$ shown in Table 1. The set of directions $D \in e^{\text {Hdirs }}$ and incoming direction $d \in$ Hdirs are together graphically denoted in a partially outlined hexagon, where marked sides correspond to elements of $D$. E.g. $D=\{\boldsymbol{\lambda}, \nearrow, \leftarrow\}, d=\searrow$ is shown as $\backslash$

Table 1. Next Position function

\begin{tabular}{|c|c|}
\hline$(\mathrm{D}, \mathrm{d})$ & $\eta_{1}(\mathrm{D}, \mathrm{d})$ \\
\hline$\pi$ & $\rightarrow$ \\
\hline$\widehat{\mapsto}$ & $\rightarrow$ \\
\hline$\nrightarrow$ & $\searrow$ \\
\hline 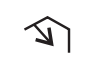 & $\searrow$ \\
\hline$\Delta$ & $\swarrow$ \\
\hline \) & $\nwarrow$ \\
\hline D) & $\nwarrow$ \\
\hline$\widehat{D}$ & $\leftarrow$ \\
\hline
\end{tabular}

\begin{tabular}{|c|c|}
\hline$(\mathrm{D}, \mathrm{d})$ & $\eta(\mathrm{D}, \mathrm{d})$ \\
\hline భ & $\leftarrow$ \\
\hline$\widehat{\leftrightarrow}$ & $\swarrow$ \\
\hline$\widehat{\imath}$ & $\rightarrow$ \\
\hline 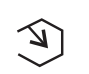 & $\leftarrow$ \\
\hline$\Leftrightarrow$ & $\nwarrow$ \\
\hline$\widehat{\leftrightarrow}$ & $\searrow$ \\
\hline $\mathbb{v}$ & $\rightarrow$ \\
\hline 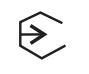 & $\rightarrow$ \\
\hline
\end{tabular}

IV. HEXAGONAL WANG AUTOMATA

We introduce hexagonal Wang automata and prove that they are equivalent to HREC.

\section{Definition 4.1}

A $\delta$--directed Hexagonal Wang automaton $(\delta-$ HWA) is a tuple $\langle\Sigma, Q, \tau, \delta, F\rangle$ where

- $\Sigma$ is a finite input alphabet
- $Q$ is a finite set of colours

- $\tau: \Sigma_{Q} \times$ Hdirs $\rightarrow 2^{\Sigma_{6 Q}}$ is a partial function such that each tile in) $\tau(A, d)$ extends $A$.

- $\delta$ is a polite scanning strategy induced by some, $\quad\left\langle\eta, \operatorname{cor}_{s}, d_{s}>\right.$ such that $\tau(A, d) \neq \phi$ implies $\gamma\left(\Delta_{A}, d\right) \neq \perp$.

- $F \subseteq \Sigma_{6 Q}$

A hexagonal Wang automaton can be viewed as having a head that visits a hexagonal picture, by moving from a position to an adjacent one and colouring at each step the edges of the position it is visiting. The elements of $E_{Q} \times$ Hdirs can be considered as states of the automaton.

For each accepting computation, the HWA produces a hexagonal Wang picture whose label is equal to the input picture. The movements of the head are lead by the scanning strategy, whereas the coloring operations of automaton are determined by $\tau$. Since hexagonal scanning strategy is polite, the automaton visits the picture positions independently of the input symbols and the choice of colors to assign to the edges is non deterministic.

\section{Example 4.1}

Consider the language $L_{b-c e n t r i c}$ presented in Example 2.4. Starting from the hexagonal Wang system developed in the same example, we define an equivalent (c) - HWA as described in Table 2. Note that $\tau(A, d)$ has at most one element for any $A, d$. The set of accepting hexagonal Wang tiles is

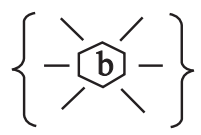

\section{Theorem 4.1}

For every polite hexagonal scanning strategy $\delta$, we have $L(\delta-H W A)=L(H T S)$.

\section{Proof}

Since HTS and HWS are equivalent [1], it is enough to prove that for every polite scanning strategy $\delta, \delta$-directed Wang automata are equivalent to hexagonal Wang systems. Let $H=<\Sigma, Q, \tau, \delta, F>$ be a $\delta$-HWA recognizing a language $L$. Then given 
$A^{\prime} \in \tau(A, d)$ let $d^{\prime \prime}=\eta\left(\Delta_{A}, d\right)$ and, for every direction $y$, set

$$
g(y)=\left\{\begin{array}{l}
d^{\prime} \text { if } y=d^{\prime} \\
d \text { if } y=-d \\
\perp \text { otherwise }
\end{array}\right.
$$

If we consider the labeled hexagonal Wang tile $C \in E_{6}(Q>$ Hdirs $)$ defined by setting and $C_{d}\left(A_{d}^{\prime}, g(d)\right)$ for any direction $d$. These labeled hexagonal Wang tiles $C$ carry two information: the colour $\mathrm{C}_{d}$ assigned by automaton through $A_{d}^{\prime}$ for the borders and the path followed by the head of the automaton, corresponding to the scanning strategy $\delta$. For every $G \in F$, consider all tiles $J \in \Sigma_{6}(Q-H$ dirs $)$ which are like $G$, but exactly one edge coloured with an inbound arrow (corresponding to current direction), while all the other edges have their second component as $\perp$. Let $\theta$ be the set of any such $C$ and $J$. It can be easily verified that each hexagonal Wang picture over corresponds to an accepting computation of HWA. Hence $L$ is the language generated by the hexagonal Wang system $W=(Q \times$ Hdirs, $\theta)$.

Conversely, let $W=(Q, \Sigma, \theta)$ be a hexagonal Wang system recognizing a language $L$. Then take any polite hexagonal scanning $\delta$ strategy and define $\delta$-HWA,

$$
H=\langle\Sigma, Q, \tau, \delta, F\rangle \text { where } F \text { is set of all }
$$
hexagonal Wang tiles over $Q$ and $\tau$ is defined by setting, for each direction $d$ and partial hexagonal tile A:

$$
\tau(A, d)=\left\{\begin{array}{lr}
\{B \in \theta / B \text { extends } A\} & \text { if } \eta\left(\Delta_{S}, d\right) \neq \perp \\
\varphi & \text { otherwise }
\end{array}\right\}
$$

It can be proved that the language generated by $H$ is $L$ and this concludes the proof.

\section{DETERMINISM IN HEXAGONAL WANG AUTOMATA}

Now we introduce the determinism in the frame work of Hexagonal Wang Automata

\section{Definition 5.1}

A $\delta$ - HWA $\langle\Sigma, Q, \tau, \delta, F\rangle$ is deterministic if $\tau(A, d)$ has at most one element for every hexagonal tile $A \in \Sigma X Q^{6}$ and direction d. Deterministic -HWA are denoted by $\delta$-DHWA. The union of classes $L$ ( $\delta$-DHWA) over all polite scanning strategies is denoted by pscan-DHREC.

If $\tau$ is the transition function of $\delta$-DHWA, we write $\tau(A, d)=B$ instead of $\tau(A, d)=\{B\}$.

\begin{tabular}{|c|c|c|c|c|c|c|c|}
\hline A & d & $\tau$ & $\eta$ & A & d & $\tau$ & $\eta$ \\
\hline $\begin{array}{c}\# \# \\
\# \text { 递 }\end{array}$ & 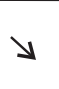 & $\begin{array}{c}\text { \#\# } \\
\text { \#的. }\end{array}$ & $\rightarrow$ & $\cdot[a]$ & $\rightarrow$ & 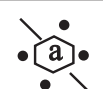 & $\rightarrow$ \\
\hline$\#$ \# & & \# \# & & & & & \\
\hline - a & $\rightarrow$ & $\stackrel{a}{a} \cdot$ & $\rightarrow$ & - a & $\rightarrow$ & $\bullet$ & $\rightarrow$ \\
\hline $\begin{array}{c}\# \# \\
\text { • (a) }\end{array}$ & $\rightarrow$ & $\begin{array}{c}\# \# \\
\text { • (a) } \#\end{array}$ & 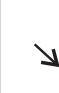 & - (a). & $\rightarrow$ & & $\searrow$ \\
\hline : \# \# & $y$ & $\cdot \stackrel{\# \#}{\cdot(a) \#}$ & ע & 悋. & $\searrow$ & 1). & $\searrow$ \\
\hline •\# & & . \# & & & & & \\
\hline (a) \# & $\nu$ & - - & $\kappa$ & (a)- & 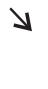 & & 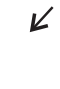 \\
\hline$\underset{\#}{\dot{a}}$ & 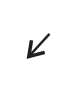 & $\cdot \stackrel{\bullet a}{a} \#$ & $\swarrow$ & & $\swarrow$ & & $\swarrow$ \\
\hline$\underset{\# \#}{\stackrel{\bullet}{a} \#}$ & $\swarrow$ & $\cdot \underset{\# \#}{\cdot a}$ & $\leftarrow$ & a). & $\swarrow$ & & $\leftarrow$ \\
\hline$\underset{\# \#}{a}$ & $\leftarrow$ & • $\underset{\# \#}{a}$ & $\leftarrow$ & a & $\leftarrow$ & & $\leftarrow$ \\
\hline 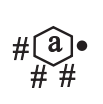 & $\leftarrow$ & \#这. & $\pi$ & a & $\leftarrow$ & & $\pi$ \\
\hline$\#$ a & $\pi$ & \#这· & $\pi$ & - a & $\pi$ & • & $\pi$ \\
\hline $\begin{array}{l}\# \bullet \\
\#\end{array}$ & & $\begin{array}{l}\# \bullet \\
\# \bullet\end{array}$ & & & & & \\
\hline \#@ & $\leftarrow$ & $\underset{\# \bullet}{\#-a-}$ & $\pi$ & & $\pi$ & & $\pi$ \\
\hline $\begin{array}{c}\# \\
\# \text { 迎 }\end{array}$ & $\pi$ & \#• & $\pi$ & - - & $\pi$ & • $\ddot{a}$ a & $\rightarrow$ \\
\hline$\ddot{\#}$ & & $\ddot{\#}$ & & $\therefore$ & & & \\
\hline \#@a & $\pi$ & $\#$ \#可. & $\rightarrow$ & a & $\downarrow$ & & $\swarrow$ \\
\hline & & & & $\because a$ & $\pi$ & & $\perp$ \\
\hline
\end{tabular}

Table 2 Computation of a Hexagonal Wang Automaton with spiral scanning strategy 
Table 3 Computation of a Deterministic Hexagonal Wang Automaton with Arrow head like scanning strategy

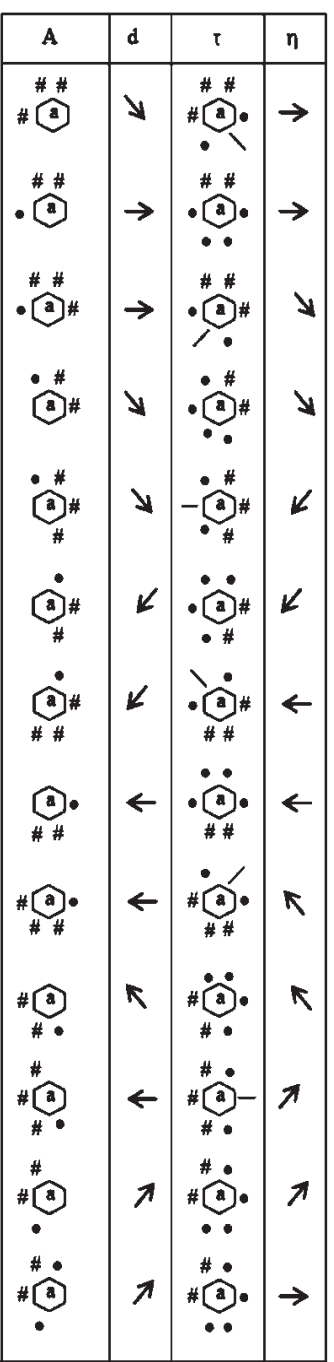

\begin{tabular}{|c|c|c|c|}
\hline A & $d$ & I & $\eta$ \\
\hline - 1 & $\rightarrow$ & • & $\rightarrow$ \\
\hline$\ddot{0}$ & $\rightarrow$ & (). & $\rightarrow$ \\
\hline - & $\rightarrow$ & & $y$ \\
\hline (). & $\forall$ & & 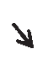 \\
\hline & $\searrow$ & & $\kappa$ \\
\hline (s) & $k$ & & $k$ \\
\hline 4 & $\swarrow$ & & $\leftarrow$ \\
\hline (7). & $\leftarrow$ & (a). & $\leftarrow$ \\
\hline (0) & $\leftarrow$ &. & $\pi$ \\
\hline 0 & $\pi$ & (a) & $k$ \\
\hline - ia & $\pi$ & & $\pi$ \\
\hline - (a) & $\pi$ & (a) & $\rightarrow$ \\
\hline (a) & $\forall$ & $\ddot{a}-$ & $\rightarrow$ \\
\hline
\end{tabular}

\section{Example 5.1}

The (a) -HWA given in Example 4.1 is

deterministic.

\section{Example 5.2}

The language $L_{x \text {-parallel }}$ given in Example 2.3 can be recognized by $\longrightarrow$-DHWA (see the Table 3).

The set of accepting tiles is

where $x \in\{a, b\}$

\section{Proposition 5.1} disjoint.

\section{Proof}

The language $L_{b \text {-centric }}$ is in $L($ (d) -DHWA) as shown in Example 5.1. This language can also be recognized by an $\longrightarrow$ - DHWA by a simple construction where the scanning strategy identifies the center $b$ once it colours the tiles of the following form in the border

\section{Proposition 5.2}

There exists a language in $L$ ( (d) -DHWA) and not in $L(\neg-D H W A)$

\section{Proof}

The language $L_{x \text {-parallel }}$ is in $L(\neg$-DHWA $)$ as shown in Example 5.2. This language cannot be recognized by an (2)- DHWA as every turn of a spiral

is able to propagate the symbol only in the first half of the hexagonal picture but not in the opposite border.

\section{Proposition 5.3}

There exists a language in $L($ (D) -DHWA) and not in $L(\neg-D H W A)$

\section{Proof}

Consider the language $\mathrm{L}_{\text {Thalf }} \subset \Sigma^{* * \mathrm{H}}$ of pictures of size $(l, m, n), l, m, n \geq 2$ and $n$ is even, with top border is of the form $x \cdot \bar{x}$ where $\bar{x}$ is the reverse of the string $x$. This language can be recognized by a (a) -DHWA $\langle\Sigma, Q, \tau, \delta, F\rangle$ The spiral scanning strategy can be devised as follows. The first spiral turn will matches the corner symbols of the top border and propagate the remaining elements downwards and check them in the second spiral turn; The rest of the computation is as before. Hence $L_{\text {Thalf }}$ is in $L(\longrightarrow$ DHWA). But this language cannot be recognized by (a)- DHWA as the scanning lacks the mechanism to propagate the information about the symbols in the palindromic positions in the top border.

The following theorem is immediate consequence of the above propositions

\section{Theorem 5.1}

L(อ-DHWA) and L $\multimap D H W A)$ are incomparable. 


\section{Vl. CONCLUSION}

Hexagonal Wang automata (HWA) can be considered as one of the natural models to recognize the hexagonal pictures of ground level class HREC. The strength of this model is combining the power of Hexagonal Wang systems with simple hexagonal scanning strategies. The polite strategies naturally induce many deterministic subclasses of HWA alternative to the traditional diagonal determinism given by hexagonal online tessellation automata. Since polite hexagonal scanning strategies do not require the content and size knowledge of hexagonal picture, the application of HWA in image analysis should be explored further.

\section{REFERENCES}

[1] Dersanambika K S, Krithivasan K, Martin-Vide C and Subramanian $\mathrm{K} \mathrm{G}$, Local and recognizable hexagonal picture languages, International Journal of Pattern Recognition and Artificial Intelligence, 19(7):853-871, 2005.

[2] Lonati $V$ and Pradella $M$, Picture recognizability with automata based on Wang tiles, In Proc. SOFSEM 2010, Vol. 5901 of LNCS, 576-587, Springer, 2010.
[3] Inoue $\mathrm{K}$ and Takanami I, A survey of two-dimensional automata theory, Information Sciences, 55(1-3):99-121, 1991.

[4] Siromoney G and Siromoney R, Hexagonal arrays and rectangular blocks, Computer Graphics and Image Processing, 5:353-381, 1976.

[5] Kamaraj T, Thomas D G and Kalyani T, Hexagonal picture recognizability by Hexagonal Wang Automata, In Proc. of ICMCM 2011, Narosa Publishing, 378-388, 2012.

T. Kamaraj received the M.Sc. degree in Mathematics, in 2000 and M.Phil. degree in 2001 from Madras University. He is at present on the teaching faculty of Department of Mathematics, Sathyabama University, Chennai. He is doing research in the areas of hexagonal array grammars and automata and partial commutation on arrays. He has published many research papers in International and national conferences and Journals. 